\title{
A Fixture Assembly Sequence Planning Method Based on Ant Colony Algorithm
}

\author{
Yan $\mathrm{CaO}^{1 \mathrm{a}}$, Zhuanxia $\mathrm{Li}^{1}$ and Sen $\mathrm{CaO}^{2}$ \\ ${ }^{1}$ School of Mechatronic Engineering, Xi'an Technological University, Xi'an, Shaanxi, China \\ ${ }^{2}$ Shandong Vocational College of Economics and Business, Weifang, Shandong, China \\ a jantonyz@163.com
}

Keywords: Fixture; Assembly sequence planning, Ant colony algorithm; Matlab.

\begin{abstract}
In order to solve the assembly sequence planning problem of fixtures, an ant colony algorithm is presented according to the principle that an assembly can be assembled if it can be disassembled. The disassembly constraint relations among the parts in a fixture are used to construct an integrated disassembly interference matrix. The key technologies are discussed, including feasible disassembly direction derivation based on an interference matrix, state transition probability of disassembly parts, pheromone updating rule of the ant colony algorithm and so on. The objective function takes the changing times of disassembly directions and tools. A program is realized in Matlab and verified by an example. The algorithm can effectively find the optimal assembly sequences for fixtures.
\end{abstract}

\section{Introduction}

In general, assembly sequence planning ${ }^{[1,2]}$ was carried out based on CAD models. First, a mathematical model for assembly sequence planning is established. Then, the model is used to represent and solve the assembly sequence planning problem. An important modeling task is to determine the mutually positional relations between the parts in the assembly. It is of great significance for various assembly sequence planning methods. In the paper, ant colony algorithm ${ }^{[3-5]}$ is applied to solve the fixture assembly sequence planning problem. It can improve assembling efficiency in enterprises, reduce assembling costs, shorten assembling cycle and enhance market competitiveness.

\section{Model of an Ant Colony Algorithm}

Determining disassembly operation sequence is based on an integrated interference matrix and state transition probability.

Integrated Interference Matrix. There cannot be interference between parts in the disassembly process of a fixture. The integrated interference matrix can easily describe the interference relation between any parts in $\pm \mathrm{X}, \pm \mathrm{Y}$ and $\pm \mathrm{Z}$ directions. Then, through the operations between the matrix elements, a feasible assembly direction set of a part in an assembly sequence can be obtained.

The integrated interference matrix of a fixture consisting of $n$ parts is as below.

$$
I_{M}=\left[\begin{array}{cccc}
I_{11 x} I_{11 y} I_{11 z} & I_{12 x} I_{12 y} I_{12 z} & \ldots & I_{1 n x} I_{1 n y} I_{1 n z} \\
I_{21 x} I_{21 y} I_{21 z} & I_{22 x} I_{22 y} I_{22 z} & \ldots & I_{2 n x} I_{2 n y} I_{2 n z} \\
\ldots & \ldots & \ldots & \ldots \\
I_{n 1 x} I_{n 1 y} I_{n 1 z} & I_{n 2 x} I_{n 2 y} I_{n 2 z} & \ldots & I_{n n x} I_{n n y} I_{n n z}
\end{array}\right]
$$

Hereinto, $I_{i j x}$ is the interference status of part $p_{j}$ along $+\mathrm{X}$ direction with part $p_{i}$ when part $p_{i}$ does not move. If there is interference, $p_{i j x}=1$. Otherwise, $I_{i j x}=0$. After a disassembly operation is 
completed, dynamically update the elements in the matrix and determine the parts that can be removed until all the parts are removed.

State Transition Probability of disassembly parts. The probability that an ant in a colony switches from one operation to another is referred to as the state transition probability. Set the number of ants to be $\mathrm{m}$. The ant switches from one operation to the next operation in terms of the probability value. Assume that the ant's path $(\mathrm{i}, \mathrm{j}$ ) is from node $\mathrm{i}$ to node $\mathrm{j}$, the state transition probability depends on the pheromone concentration $\tau_{i j}$ on the path, direction guide factor $d_{i j}$, tool guide factor $t_{i j}$ jointly. The greater $d_{i j}$ and $t_{i j}$, the greater disassembly transition probability, the more likely the ant chooses the path. Transition probability $p_{i j}^{k}(t)$ is defined as follows.

$$
p_{i j}^{k}(t)=\left\{\begin{array}{cc}
\frac{\left[\tau_{i j}(t)\right]^{\alpha}\left[d_{i j}(t)\right]^{\beta}\left[t l_{i j}(t)\right]^{\mu}}{\sum_{s \in \text { allowed }_{k}}\left[\tau_{i k}(t)\right]^{\alpha}\left[\eta_{i k}(t)\right]^{\beta}\left[t l_{i k}(t)\right]^{\mu}}, j \in s \in \text { allowed }_{k} \\
0 & \text { others }
\end{array}\right.
$$

Hereinto, allowed $_{k}$ is the allowed disassembly operations that the ant can chooses next. $\tau_{i j}(t)$ is the residual pheromone between operation $\mathrm{i}$ and operating $\mathrm{j}$ at time $\mathrm{t}$. $d_{i j}(t)$ is the disassembly direction change factor between operation $i$ and operating $j$ at time $t$. Its value is in span $(0,1)$. If the disassembly direction changes, it takes a smaller value in the span $(0,1)$. Otherwise, it takes a greater value in the span $(0,1) \cdot t_{i j}(t)$ is the disassembly tool change factor between operation $\mathrm{i}$ and operating $\mathrm{j}$ the at time t. Its value is in span $(0,1)$. If the disassembly tool changes, it takes a smaller value in the span $(0,1)$. Otherwise, it takes a greater value in the span $(0,1) . \alpha$ is an information heuristic factor. $\beta$ and $\mu$ are expectation heuristic factors. All the three factors are nonnegative numbers that represent the degree of effect on the state transition probability.

Pheromone update of the ant colony algorithm. After all the ants complete a cycle, the pheromone on all the edge reduces according to a certain ratio. Then, the pheromone on the edges passed by the ants in the current cycle increases. The increased amount of pheromone is determined by the quality evaluation of the path.

After all the ants complete a cycle, the pheromone updating rule of the edges is as below.

$$
\tau_{i j}(t+1)=(1-\rho) \tau_{i j}(t)+\sum_{k=1}^{m} \Delta \tau_{i j}^{k}(t) .
$$

Hereinto, $\rho$ is the degree of pheromone volatilization from time t to time $(\mathrm{t}+1), \Delta \tau_{i j}$ is the increment of pheromone of ant k on path $(\mathrm{i}, \mathrm{j})$ in the current cycle, and $\Delta \tau_{i j}^{k}(t)$ can be obtained with Eq. 4 where $Q$ is pheromone strength and $f$ is the value of the objective function.

$$
\Delta \tau_{i j}^{k}=\left\{\begin{array}{rr}
\frac{Q}{f} & \text { If ant k passes path (i, } \mathrm{j}) \\
0 & \text { others }
\end{array} .\right.
$$

Establishment of the objective function. Taking the changing number of reorientation and disassembly tools as the goal, the objective function of assembly sequence planning is as below.

$$
f=w_{1} * D+w_{2} * T \text {. }
$$

Hereinto, $D$ is the number of disassembly direction changes, $T$ is the number of disassembly tool changes, and $w_{1}$ and $w_{2}$ are their weight coefficients.

The less the number of disassembly direction changes and tool changes, the less the objective function, and the better the quality of the assembly sequence. 


\section{Procedure of the Ant Colony Algorithm to Optimize Assembly Sequence}

The ant colony algorithm for fixture assembly sequence optimization is realized in Matlab. The detailed procedure is as follows.

Step 1: Initialize the value of each parameter. According to the integrated disassembly matrix of an assembly, generate the initial feasible disassembly operations. Randomly distribute the ants on these nodes.

Step 2: For the choice scope of each ant, calculate the value of the state transition probability formula respectively. According to the size of the state transition probability, choose the next disassembly operation.

Step 3: After each selection, dynamically simplify the integrated interference matrix and compute the next optional disassembly operation scope.

Step 4: Judge whether the number of all the removed parts is equal to that of all the parts and sub-assemblies in the assembly. If not, repeat steps 2 and 3. Otherwise, go to the next step.

Step 5: In terms of the objective function, save the current optimal assembly sequence.

Step 6: According to the quality of the solutions, update the global pheromone, and go to the next cycle.

Step 7: Judge whether the number of cycles is equal to the maximum cycle number. If not, repeat steps 5 and 6 . Otherwise, end the algorithm and output the optimal assembly sequence.

\section{An Example and its Optimization Results}

Taking a lathe fixture for example, use the ant colony algorithm to optimize its assembly sequence. Its three-dimensional model is shown in Fig. 1. Hereinto, 1 is an adjusting screw, 2 is a guide block, 3 and 4 are bolts, 5 is a directional block, 6 and 7 are positioning pins, 8 is a locating block, 9 is a clamping strip, 10 are nut, gasket and bolt, 11 is a spring, 12 is a support bar and 13 is the fixture main body.

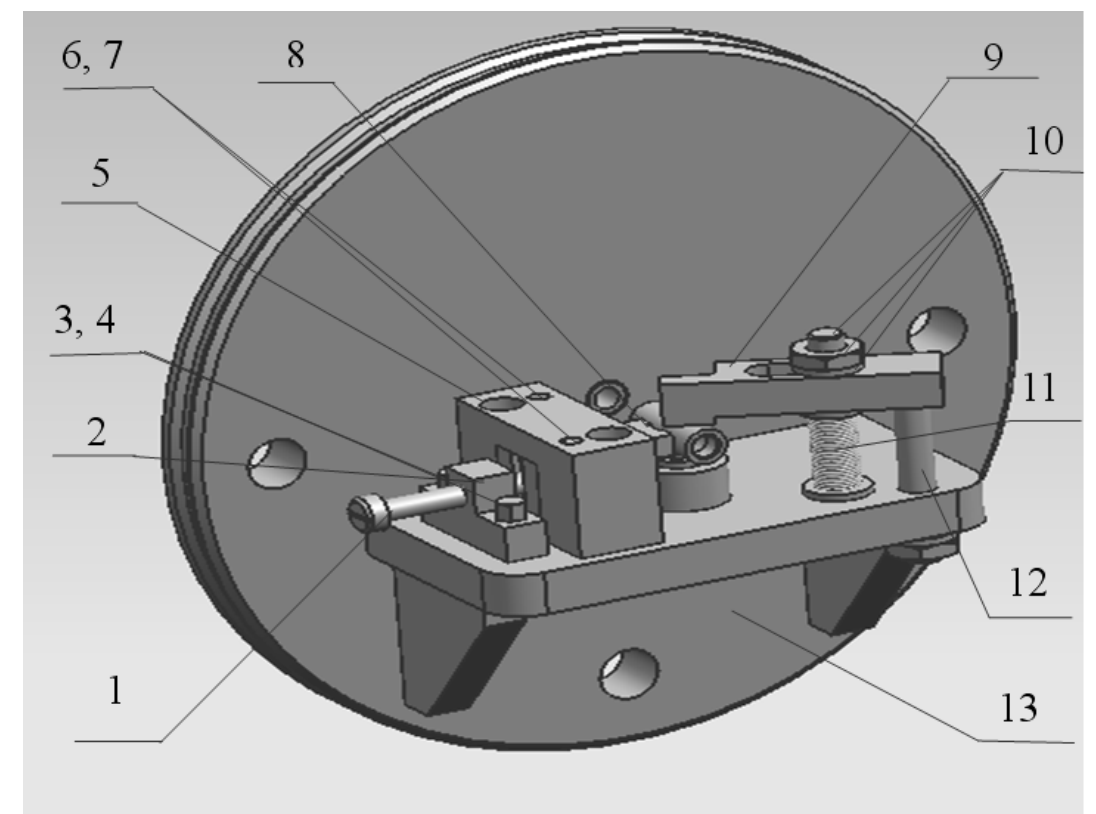

Figure 1 The three-dimensional model of the lathe fixture

Run the algorithm in Matlab to get the optimal assembly sequence of the lathe fixture. The convergence curve of the algorithm is shown in Fig. 2. The red line is the relation between the number of iterations and the average value of the objective function. The blue line is the relation between the number of iterations and the optimal value of the objective function.

The optimal disassembly sequence of the lathe fixture is $3,4,2,1,6,7,5,8,10,9,11,12$ and 13 . 


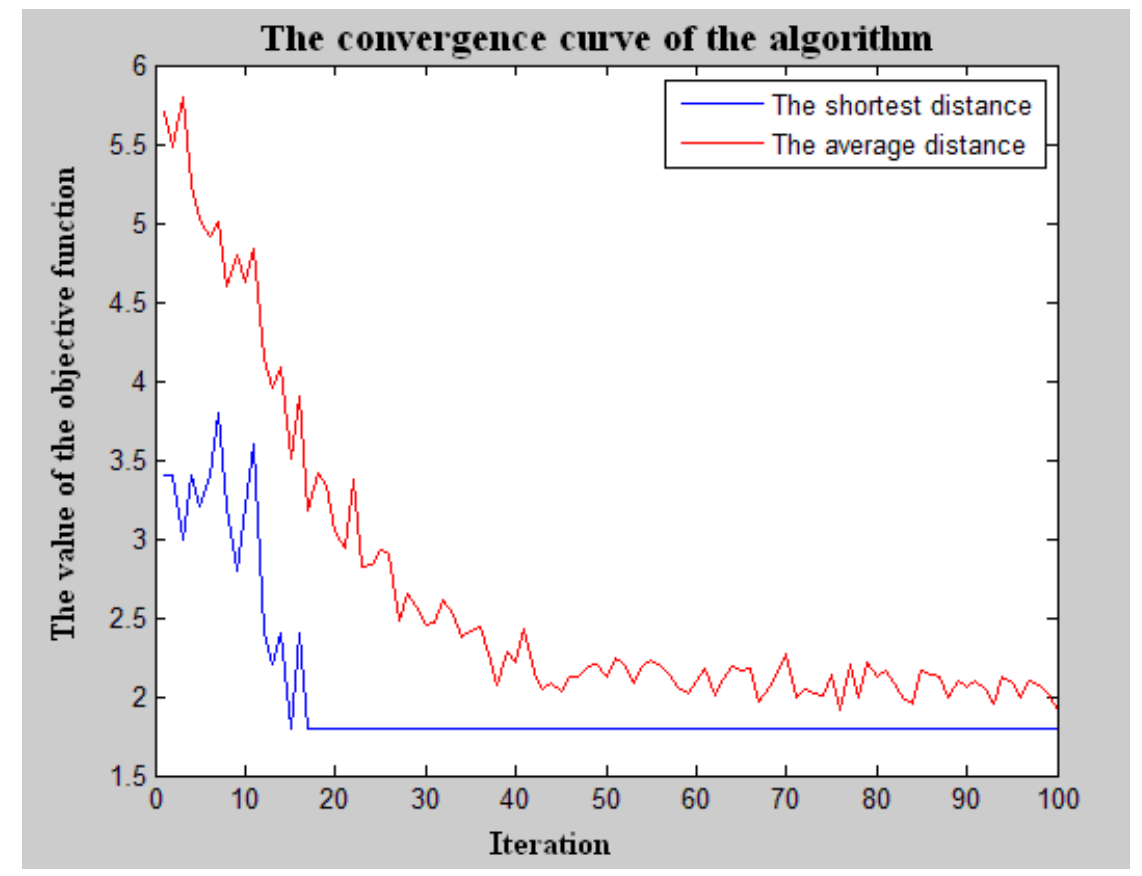

Figure 2 The convergence curve of the algorithm

\section{Conclusions}

In traditional fixture assembling process, engineers rely on their knowledge and experience in most companies to complete assembly sequence planning. But it is very difficult to find a relatively optimal assembly sequence in a short time when there are so many parts in an assembly and you have to make a good assembly sequence only with your own knowledge and experience. In the paper, the ant colony algorithm is used to find out the optimal assembly sequence. The algorithm is realized in Matlab and verified by an example.

\section{Acknowledgments}

The work is supported by Open Research Fund Program of Shaanxi Key Laboratory of Non-Traditional Machining, Key Problem Tackling Project of Shaanxi Scientific and Technological Office (2012K06-19), 2011 Key Education Innovation Project of Xi'an Technological University (11JGZ02), Natural Science Basic Research Plan in Shaanxi Province of China (2013JM7029) and Shaanxi Major Subject Construction Project.

\section{References}

[1] Q. Su: Robotics and Computer-Integrated Manufacturing Vol. 25 No. 1 (2009), p. 224

[2] N. Ye, P. Banerjee, A. Banerjee and F. Dech: IEEE Transaction on Systems, Man, and Cybernetics Parts C Vo1. 29 No. 4 (1999), p. 546

[3] F. Failli and G. Dini: Proceeding of the 2nd CIRP International Seminar in Intelligent Computation in Manufacturing Engineering (2000), p. 227

[4] H.B. Duan, D.B. Wang and X.F. Yu: Journal of Communication and Computer Vol. 2 No. 9 (2005), p. 32

[5] P.R. McMullen: Articial Intelligence in Engineering Vol. 15 No. 3 (2001), p. 309 